\title{
REAL-TIME ON-ORBIT CALIBRATION OF ANGLES BETWEEN STAR SENSOR AND EARTH OBSERVATION CAMERA FOR OPTICAL SURVEYING AND MAPPING SATELLITES
}

\author{
Wei Liu ${ }^{1 *}$, Hui Wang ${ }^{2}$, Weijiao Jiang ${ }^{2}$, Fangming Qian ${ }^{3}$, Leiming Zhu ${ }^{4}$ \\ ${ }^{1}$ Xi'an Research Institute of Surveying and Mapping, No.1 Middle Yanta Road, Xi'an, China - 1271772072@qq.com \\ ${ }^{2}$ State Key Laboratory of Integrated Service Network, Xidian University, Xi'an, China - huiwang_ii@yeah.net \\ ${ }^{2}$ State Key Laboratory of Integrated Service Network, Xidian University, Xi'an, China - 1510142681@qq.com \\ ${ }^{3}$ Information Engineering University, Zhengzhou, China -122748721@qq.com \\ ${ }^{4}$ Centre of TH-Satellite of China, Beijing, China - zlmwgf@qq.com
}

Commission I, WG I/4

KEY WORDS: Star Sensor, Attitude Measurement, Error Analysis, On-Orbit Calibration, Angle between Cameras, On-orbit Monitoring Device

\begin{abstract}
:
On space remote sensing stereo mapping field, the angle variation between the star sensor's optical axis and the earth observation camera's optical axis on-orbit affects the positioning accuracy, when optical mapping is without ground control points (GCPs). This work analyses the formation factors and elimination methods for both the star sensor's error and the angles error between the star sensor's optical axis and the earth observation camera's optical axis. Based on that, to improve the low attitude stability and long calibration time necessary of current satellite cameras, a method is then proposed for real-time on-orbit calibration of the angles between star sensor's optical axis and the earth observation camera's optical axis based on the principle of auto-collimation. This method is experimentally verified to realize real-time on-orbit autonomous calibration of the angles between the star sensor's optical axis and the earth observation camera's optical axis.
\end{abstract}

\section{INTRODUCTION}

Exterior orientation elements are a key factor influencing the positioning accuracy, when performing satellite photogrammetry without ground control points (GCPs) (Wang, 2004). Exterior orientation elements are commonly determined by converting the star sensor's measured attitude, using the angle conversion relationship between the star sensor's optical axis and the earth observation camera's optical axis. However, the accuracy of the exterior orientation elements is affected by systematic or random errors in both the star sensor and the angles between the star sensor's optical axis and the earth observation camera's optical axis contain, caused by various on-orbit factors, such as pressure, vibration and temperature. Based on previous research results and a large amount of measured data we've accumulated in the long-term, the paper analyses the measurement error and on-orbit calibration results of the star sensor, and finds that the thermo elastic error is the main factor to cause the angle variation between the star sensor's optical axis and the earth observation camera's optical axis, which limits the positioning accuracy. Currently, two methods are used to address the angles error. In one, the structure is optimized and the heat-resistant material is used during load development (Blarre et al., 2006). However, due to factors such as temperature control level and technological level, this method has only offered a limited improvement on positioning accuracy. In the other, a photographic calibration method based on the ground calibration field is used after the satellite is on-orbit (Radhadevi et al., 2011), (Chen et al., 2015). However, the calibration period of the existing calibration method will not be able to meet the needs of future high-precision surveying and mapping capabilities. Therefore, an efficient and high-precision real-time calibration method for the angles between the star sensor's optical axis and the earth observation camera's optical axis is needed.

Therefore, this paper presents a real-time calibration method of the angles between the star sensor's optical axis and the earth observation camera's optical axis. The method can be realized on the optical mapping satellite on-orbit by installing an optical autocollimator inside the star sensor and earth observation camera optical systems. The change of the main optical axis of each camera can be reflected by the change of the spot image position, using the original optical path of the camera. Subsequently, we calculate the angular variation between the star sensor's optical axis and the earth observation camera's optical axis, allowing for real-time calibration on the satellite.

\section{ATTITUDE MEASUREMENT PRINCIPLE}

\subsection{Attitude Measurement Principle of Star Sensor}

The star sensor is a high-precision space attitude measuring device used to determine the three-axis attitude of a satellite, which uses stars as reference objects. The instantaneous pointing of the star sensor in the inertial coordinate system is calculated using a photographed stellar image. Then, the exterior orientation elements of the earth observation camera are calculated according to the angles between the star sensor's optical axis and the earth observation camera's optical axis. The working principle of the star sensor can be divided into star extraction (Delabie, Schutter and Vandenbussche, 2014), star identification (Padgett, Kreutz-Delgado and Udomkesmalee, 2012), and

\footnotetext{
* Corresponding author
} 
attitude calculation (Psiaki and Mark, 2000), as shown in Figure 1 .

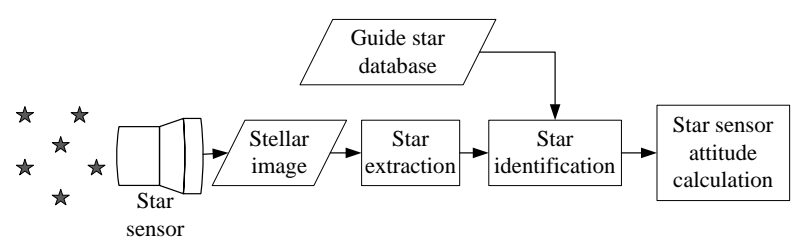

Figure 1. Working principle of star sensor

The attitude parameters of the satellite have various expressions, such as direction cosine, Euler angles (attitude angles), and quaternion. Quaternion is always used to avoid singularity in the attitude calculation. However, as the attitude angles are more intuitive and easier to understand, it is commonly used to give research indicators and rules. The conversion relationship of the attitude quaternion $\mathrm{q}_{0}, \mathrm{q}_{1}, \mathrm{q}_{2}, \mathrm{q}_{3}$ ( $\mathrm{q}_{0}$ is a scalar) to the attitude angles $\beta, \alpha$ and $\gamma$ (roll, pitch, and yaw) (rotating around Z-axis, then $\mathrm{X}$-axis, at last $\mathrm{Y}$-axis) is:

$$
\left[\begin{array}{l}
\alpha \\
\beta \\
\gamma
\end{array}\right]=\left[\begin{array}{c}
\tan ^{-1} \frac{2\left(q_{1} q_{3}-q_{0} q_{2}\right)}{1-2\left(q_{0}^{2}+q_{3}^{2}\right)} \\
\sin ^{-1}\left[2\left(q_{2} q_{3}+q_{0} q_{1}\right)\right] \\
\tan ^{-1} \frac{2\left(q_{1} q_{2}-q_{0} q_{3}\right)}{1-2\left(q_{0}^{2}+q_{2}^{2}\right)}
\end{array}\right]
$$

2.2 Calculation of the exterior orientation elements of the earth observation camera

The star sensor measures the attitude of the star sensor's coordinate system relative to the inertial coordinate system. Here, the angles between the star sensor's optical axis and the earth observation camera's optical axis is used to establish a relationship between the star sensor's coordinate system and the earth observation camera's coordination system to then calculate the attitude of the earth observation camera relative to the inertial coordinate system.
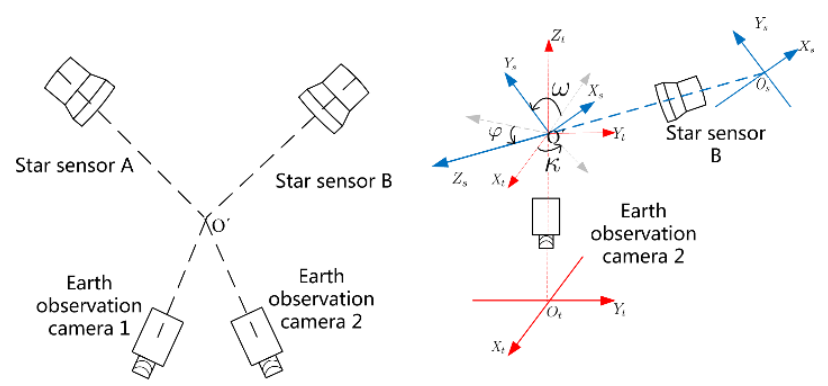

$\mathrm{O}_{\mathrm{S}}-\mathrm{X}_{\mathrm{S}} \mathrm{Y}_{\mathrm{S}} \mathrm{Z}_{\mathrm{S}}$ - star sensor measuring coordinate system

$\mathrm{O}_{\mathrm{t}}-\mathrm{X}_{\mathrm{t}} \mathrm{Y}_{\mathrm{t}} \mathrm{Z}_{\mathrm{t}}$ - earth observation camera coordination system

$\left(\begin{array}{lll}\varphi & \omega & \kappa\end{array}\right)$ - angles between the star sensor's optical axis and the earth observation camera's optical axis

Figure 2. Angles between the star sensor's optical axis and the earth observation camera's optical axis

Figure 2 shows the angles between the star sensor's optical axis and the earth observation camera's optical axis. The transformation matrix of the star sensor's coordinate system in the earth observation camera's measuring coordinate system $M_{s}^{t}$ is (rotating around $\mathrm{Z}$-axis, then $\mathrm{X}$-axis, at last $\mathrm{Y}$-axis) (Wang, 1990):

$$
\begin{aligned}
M_{s}^{t} & =\left[\begin{array}{ccc}
\cos \varphi & 0 & -\sin \varphi \\
0 & 1 & 0 \\
\sin \varphi & 0 & \cos \varphi
\end{array}\right]\left[\begin{array}{ccc}
1 & 0 & 0 \\
0 & \cos \omega & \sin \omega \\
0 & -\sin \omega & \cos \omega
\end{array}\right]\left[\begin{array}{ccc}
\cos \kappa & \sin \kappa & 0 \\
-\sin \kappa & \cos \kappa & 0 \\
0 & 0 & 1
\end{array}\right] \\
& =\left[\begin{array}{ccc}
\cos \varphi \cos \kappa-\sin \varphi \sin \omega \sin \kappa & \cos \varphi \sin \kappa+\sin \varphi \sin \omega \cos \kappa & -\sin \varphi \cos \omega \\
-\cos \omega \sin \kappa & \cos \omega \cos \kappa & \sin \omega \\
\sin \varphi \cos \kappa+\cos \varphi \sin \omega \sin \kappa & \sin \varphi \sin \kappa-\cos \varphi \sin \omega \cos \kappa & \cos \varphi \cos \omega
\end{array}\right]
\end{aligned}
$$

Then:

$$
M_{t}^{g}=M_{s}^{g}\left(M_{s}^{t}\right)^{-1}=\left[\begin{array}{lll}
A_{11} & A_{12} & A_{13} \\
A_{21} & A_{22} & A_{23} \\
A_{31} & A_{32} & A_{33}
\end{array}\right]
$$

Where $M_{s}^{g}=$ transformation matrix of the star sensor's coordinate system in the inertial coordinate system calculated by the star sensor's attitude $\left(\beta_{s}, \alpha_{s}, \gamma_{s}\right)$ (rotating around Z-axis, then $\mathrm{X}$-axis, at last $\mathrm{Y}$-axis)

$M_{t}^{g}=$ transformation matrix of the earth observation camera's coordinate system in the inertial coordinate system calculated by the earth observation camera's attitude $\left(\beta_{t}, \alpha_{t}, \gamma_{t}\right)$ (rotating around $\mathrm{Z}$-axis, then $\mathrm{X}$-axis, at last $\mathrm{Y}$-axis)

$A_{i j}=$ the elements of the $\mathrm{i}$-th row and the $\mathrm{j}$-th column of the matrix $M_{t}^{g}$

Therefore, the exterior orientation elements $\left(\beta_{t}, \alpha_{t}, \gamma_{t}\right)$ of the earth observation camera can be calculated as:

$$
\left[\begin{array}{c}
\alpha_{t} \\
\beta_{t} \\
\gamma_{t}
\end{array}\right]=\left[\begin{array}{c}
-\arctan \left(\frac{A_{13}}{A_{33}}\right) \\
\arcsin \left(\mathrm{A}_{23}\right) \\
-\arctan \left(\frac{A_{21}}{A_{22}}\right)
\end{array}\right]
$$

\section{ANALYSIS OF THE ANGLES ERROR BETWEEN THE STAR SENSOR'S OPTICAL AXIS AND THE EARTH OBSERVATION CAMERA'S OPTICAL AXIS}

The stable star sensor data of the TH-1 02 satellite (Wang and HU, 2012) and calibration result of angles between the star sensor's optical axis and the earth observation camera's optical axis based on the ground calibration field was selected for statistical analysis.

\subsection{Error Statistics of Star Sensor Measurement}

The single-star sensor attitude difference method (WANG and LI, 2012), (LI and LI, 2012) was performed on fourteen randomly selected data sets of star sensor A and B in the period of 20122016. The resulting standard deviation and total error curve is shown in Table 1 and Figure 3, respectively.

The actual on-orbit measurement error of the star sensor was between 4.83" and 9.02" (X/Y axis overall), higher than the nominal value of $5^{\prime \prime}$, basically. This was likely caused because the star sensor is also subject to error factors other than the nominal error when running on orbit. We will discuss the error factors in section 3.3. 


\begin{tabular}{|c|c|c|c|c|c|c|}
\hline \multirow{2}{*}{ Date } & \multicolumn{3}{|c|}{ Total error of star sensor $\mathrm{A}\left({ }^{\prime \prime}\right)$} & \multicolumn{3}{c|}{ Total error of star sensor $\mathrm{B}(")$} \\
\cline { 2 - 7 } & $\mathrm{X}$ axis & $\mathrm{Y}$ axis & $\mathrm{Z}$ axis & $\mathrm{X}$ axis & Y axis & $\mathrm{Z}$ axis \\
\hline $2012 / 05 / 20$ & 9.4 & 2.7 & 83.2 & 5.1 & 7.9 & 72.5 \\
\hline $2012 / 09 / 03$ & 8.4 & 1.8 & 61.4 & 4.1 & 7.5 & 48.7 \\
\hline $2012 / 12 / 02$ & 9.5 & 2.8 & 56.5 & 2.9 & 8.1 & 44.3 \\
\hline $2013 / 01 / 03$ & 12.3 & 3.4 & 67.4 & 4.5 & 9.2 & 70.9 \\
\hline $2013 / 05 / 03$ & 8.2 & 1.9 & 63 & 4.9 & 7.2 & 73 \\
\hline $2013 / 10 / 23$ & 8.5 & 2.9 & 61.6 & 5.2 & 9.9 & 60.1 \\
\hline $2014 / 01 / 26$ & 10.8 & 3.9 & 68.7 & 4.2 & 8 & 68.7 \\
\hline $2014 / 05 / 22$ & 7.9 & 2.6 & 75.7 & 4.6 & 7.9 & 62.7 \\
\hline $2014 / 08 / 24$ & 9.9 & 4.7 & 68.1 & 4 & 7.4 & 52.1 \\
\hline $2015 / 02 / 18$ & 11.6 & 3.6 & 79.1 & 4.4 & 7.4 & 47.8 \\
\hline $2015 / 10 / 12$ & 8.6 & 3.3 & 65.5 & 5.2 & 8.9 & 51.8 \\
\hline $2016 / 03 / 21$ & 11.2 & 4.6 & 87.5 & 3.2 & 6.3 & 40.2 \\
\hline $2016 / 04 / 11$ & 10.2 & 3.1 & 71.2 & 3.7 & 6.6 & 40.2 \\
\hline $2016 / 10 / 26$ & 9.4 & 3.7 & 75.2 & 3.9 & 5.6 & 40.6 \\
\hline
\end{tabular}

Table 1. Standard deviation using single star sensor attitude difference method

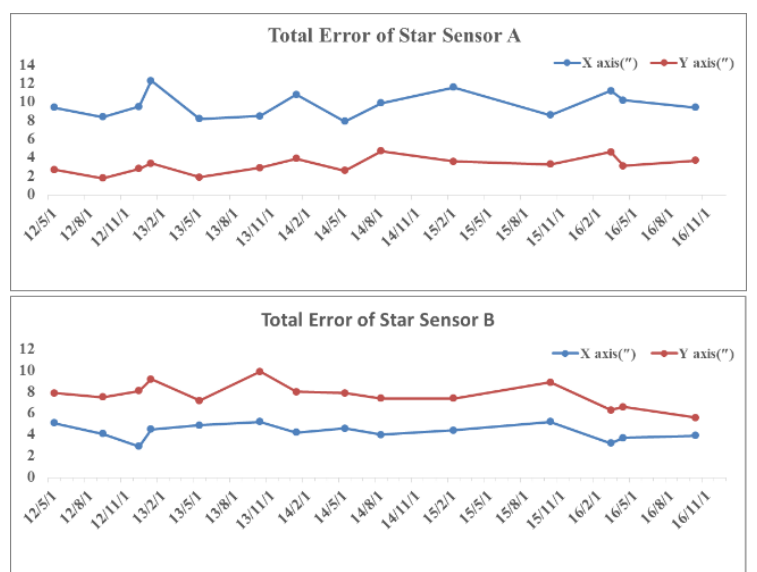

Figure 3 Total error curve of star sensor A and B

\subsection{Error Statistics Based on the Ground Calibration Field}

Based on the ground calibration field, the curve of angles errors between the star sensor's optical axis and the earth observation camera's optical axis from May 20, 2012 to December 14, 2016 of the TH-1 02 satellite is shown in Figure 4.

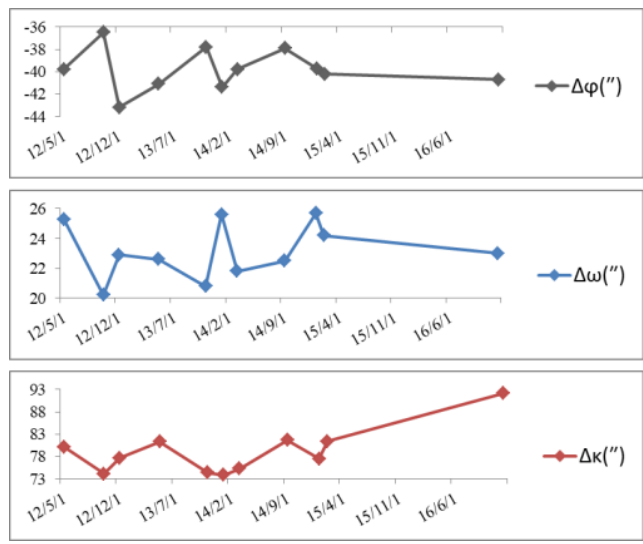

Figure 4. The calibration result of angles between the star sensor's optical axis and the earth observation camera's optical axis based on ground calibration field
Over the course of the 4.5 years, the change of the angles was a disordered, slow drifting process, making the law of change difficult to determine. Although the ground calibration method can be used to calculate angular change, the current calibration period of several months cannot be used to accurately determine short-term changes, limiting the improvement of positioning accuracy.

\subsection{Error Analysis}

Based on the error results presented in section 3.1 and 3.2 in conjunction with the general performance standard ECSS-E-ST60-20C (ECSS Secretariat, 2008) developed by the European Space Agency (ESA) in 2008, the star sensor error can be divided into temporal error (TE), high frequency error (HFE), low frequency error (LFE), and bias error (BE) according to the frequency characteristics, as shown in Figure 5 (LU et al., 2014).

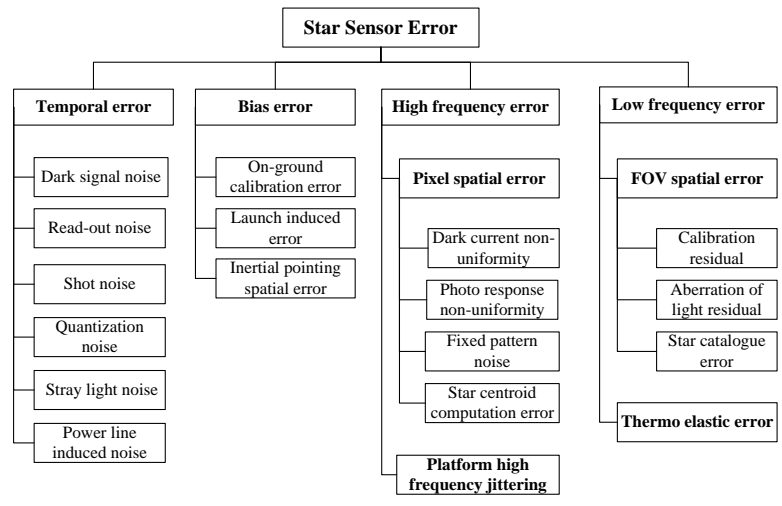

Figure 5. Breakdown of star sensor error

$\mathrm{TE}$ is a random error caused by hardware circuit noise and input signal conditions, is independent of spatial motion, and appears as irrelevant white noise in the time domain. BE refers to the inherent deviation of the star sensor's coordinate system relative to the installed coordinate system caused by environmental factors such as mechanical vibration and gravity. As a satellite should not experience mechanical shock once running on orbit, $\mathrm{BE}$ can be assumed constant. HFE manifests as pixel spatial errors and platform jittering. Pixel spatial error is due to the spatial inhomogeneity of the detector and the error caused by the 
star position. Platform jittering is caused mainly by satellite orbit maneuvering, hot and cold alternation, or the periodic movement of the moving parts on the satellite (XU et al., 2016).

The LFE an important type of regularity error that changes periodically with the satellite orbit and is mainly caused by field of view (FOV) spatial error and thermo elastic error. Japan's ALOS (Iwata et al., 2007) and ESA's Sentinel-2 (Winkler, Wiedermann and Gockel, 2008) satellites have found the existence of LFE from star sensor measurement.

Platform high-frequency jittering can be suppressed and eliminated by:

1) detecting and compensating the attitude jittering by parallax imaging based on the satellite imaging data (Amberg et al., 2013), (JIANG et al., 2014),

2) using high-frequency high-precision detection equipment to measure the jittering of the satellite platform directly (WANG et al., 2017),

3) developing a momentum wheel based on magnetic levitation (Hashimoto et al., 2013), or

4) researching the "Ultra Quiet Platform" (Fausz et al., 2012), (Erwin et al., 2013).

TE, BE, and pixel and FOV spatial errors could also be suppressed and compensated by including them in and improving the accuracy of the nominal model (Ting, Fei and Zheng, 2013) or by using more accurate calibration equipment such as a multispectral star simulator.

The above errors can be solved effectively. However, according to the existing methods, the thermo elastic error is difficult to eliminate. This paper focuses on thermo elastic error.

When the satellite is on orbit, it is affected by a variety of factors, including the orbital thermal environment, the boundary condition of the mounting surface, the temperature gradient, and the degradation of focal plane material. These factors can cause thermo elastic error of the star sensors, the earth observation cameras, and their installation structure. Here, the orbital thermal environment is the dominant error source. Due to the temperature difference between the positive and shadow areas, the camera barrel and the internal optical components produce asymmetric thermal deformation, causing mirror tilt and leading to the axis change of cameras and eventually changes to the angles between the star sensor's optical axis and the earth observation camera's optical axis (LIU et al., 2009).

The current method to suppress thermo elastic error involves onorbit design control. Composite materials with a low thermal expansion can be used to optimize the optical and mechanical structure for better integration and iso-thermalization, thereby improving the thermal stability of the star sensor and installation structure. However, due to current manufacturing technological restrictions, achieving the desired temperature and temperature control levels is difficult, making the suppression and elimination of thermo elastic error difficult in turn.

\section{OPTICAL SURVEYING AND MAPPING SATELLITE CAMERA CALIBRATION ON THE CAMERA ANGLES ON-ORBIT}

Therefore, this study proposes a real-time calibration method that applies the optical auto-collimation principle to on-orbit calibration to monitor the changes in angles between the cameras optical axes. By installing collimated light sources, area-array CCDs, prisms, and spot recording devices inside the satellite load system, changes in the cameras' optical axes can be converted into changes in the spot images. By extracting and processing the spot images, the angle variation between the cameras' optical axes can then be solved to achieve fast and efficient on-orbit calibration.

\subsection{The monitoring principle of a single camera optical axis}

The optical auto-collimation principle is a general method for measuring a bending angle. When the normal and optical axis of the reflector is at a certain angle, the deflection angle of light passing through the reflector is twice the inclination of the reflector and the offset of the imaging and light emission point of light is given by (QIAO, HE and WEI, 2012):

$$
s=f \tan 2 \theta
$$

where $f$ is the effective focal length. Therefore, the inclination angle $\theta$ can be obtained by the measuring the offset $s$.

The monitoring principle and expression of a single camera's optical axis has been analysed and derived (LIU et al., 2018); Figure 6 shows a simplified schematic of the principle.

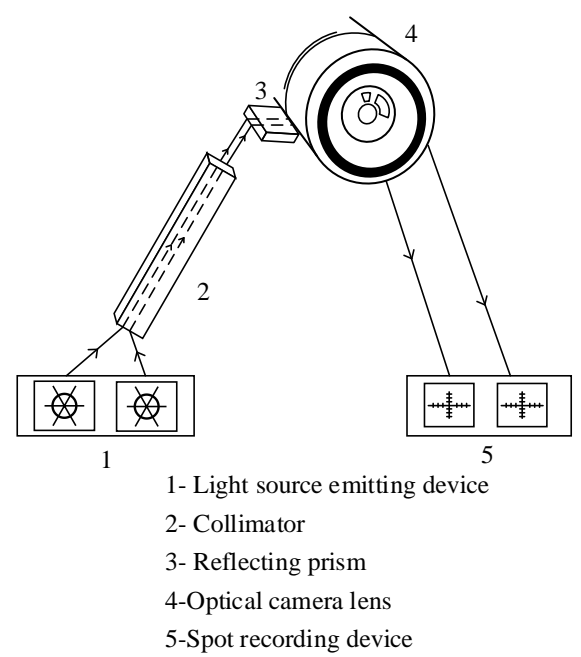

Figure 6. Schematic of the monitoring principle of a single camera's optical axis

The laser-emitting device emits a light source that is transmitted through a parallel light pipe (a light-guiding prism) to the reflecting surface of the reflecting prism. After the light is emitted onto the camera lens, it is reflected and transmitted through the optical system of the lens before being imaged on the spot recording device. The position of spot images varies with the camera's optical axis, so the changes of camera's optical axis can be derived via calculating the distance between the rated spot position and the actual position.

The deflection of the optical axis can be decomposed as follows: in the self-image space coordinate system, the optical axis is rotated by a certain $\Delta \beta$ around the $\mathrm{X}$-axis, a certain $\Delta \alpha$ around the $\mathrm{Y}$-axis, and a certain $\Delta \gamma$ around the visual axis. Simultaneously, this method can also reflect the change of camera focal length $\Delta f$. Considering the optical axis around the $\mathrm{X}$-axis as an example, the changes in spot positions are shown in Figure 7.

After derivation, the $\Delta \beta$ and $\Delta f$ are found to cause a change in the Y-coordinate of the spot image and the $\Delta \alpha$ and $\Delta \gamma$ were found to cause a change in the $\mathrm{X}$-coordinate. Therefore, as Table 2 shows, the corresponding expressions according to an integrated on-orbit variation of a single camera's optical axis are obtained, where $\delta$ is the pixel size, $f$ is the effective focal length, $Y_{M}$ and $Y_{N}$ are the Y-changes in the right and left area-arrays, respectively, $X_{M}$ and $X_{N}$ are the $\mathrm{X}$-changes in the right and left area-arrays, respectively. 


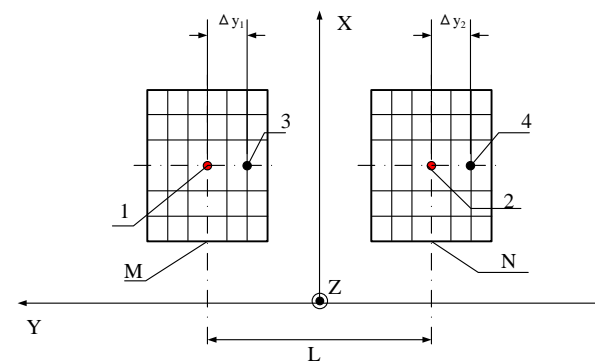

1、2-Rated imaging positions

3、4-Actual imaging positions

M、 N-Spot imaging area-arrays

$\mathrm{L}$ - The distance between the two area-array centers

Figure 7. Changes in spot positions when the optical axis rotates around the $\mathrm{X}$-axis
Verification experiments on the on-orbit calibration principle of a single camera indicated average accuracies of $\Delta \beta, \Delta \alpha$, and $\Delta \gamma$ of $0.55^{\prime \prime}, 0.64 "$, and $0.97^{\prime \prime}$ respectively, using optical autocollimations with an accuracy of $\pm 1 "$. Thus, the feasibility of the on-board monitoring method was preliminarily verified. Prior prototyping employed an off-axis three-dimensional stereo mapping camera with on-board monitoring equipment to analyse and calculate the error term. The rotation errors of visual axes around the $\mathrm{X}$ - and $\mathrm{Y}$-axis were less than $0.1^{\prime \prime}$, but the error around the Z-axis was about 2" (WANG et al., 2018). However, the error around the $\mathrm{Z}$-axis has little effect on the positioning accuracy, and could be eliminated by subsequent photogrammetric processing. The error of the change in focal length was found to be $0.04 \mathrm{~mm}$. Although the accuracy of focal length is not as good as the method based on the ground calibration field, it can be used as an initial value. Therefore, the proposed method offers a strong advantage for the measurement of the rotation of the angle.

\begin{tabular}{|c|c|}
\hline Parameters & Derived expression of uncertain changes \\
\hline Visual axis rotation around the X-axis (radian) & $\Delta \beta=\left(\frac{Y_{M}+Y_{N}}{2}\right) \cdot \frac{\delta \cos ^{2} \beta}{f}$ \\
\hline Visual axis rotation around the Y-axis (radian) & $\Delta \alpha=\left(\frac{X_{M}+X_{N}}{2}\right) \cdot \frac{\delta \cos ^{2} \alpha}{f}$ \\
\hline Camera rotation around the visual axis (radian) & $\Delta \gamma=\left(\frac{X_{M}+X_{N}}{L}\right) \cdot 2 \delta$ \\
\hline Change of focal length (mm) & $\Delta f=\left(\frac{Y_{L}-Y_{R}}{2}\right) \cdot \frac{\delta f}{L}$ \\
\hline
\end{tabular}

Table 2. Expressions of parameters

\subsection{Calibration scheme of the optical axis angle}

According to the method described in Section 4.1, the change in the optical axis of each camera is then determined by performing on-orbit monitoring of the optical axes of the star and earth cameras. Then, the changes in angles of the cameras' optical axes can be obtained, combining with the conversion relationship between the star and earth cameras derived in Section 2.2. Figure 8 shows the data processing flow.

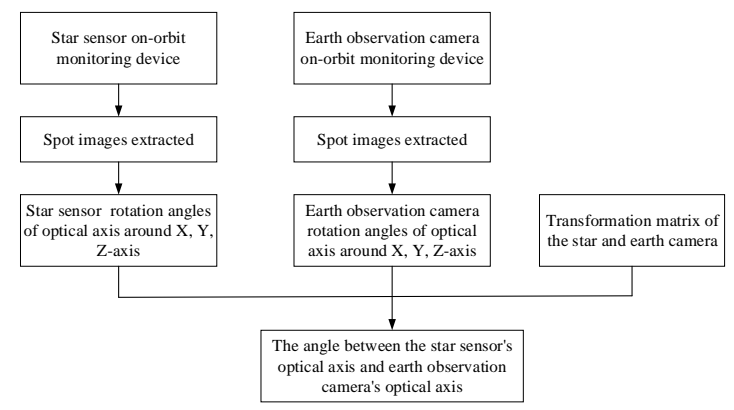

Figure 8. Data processing flow of the angle between the star senor's optical axis and earth camera's optical axis

Equations (2) and (3) can then be used to derive the changed transformation matrix of the star sensor's coordinate system in the earth observation camera's measuring coordinate system:

$$
\begin{aligned}
& M\left(\varphi_{0}+\Delta \varphi, \omega_{0}+\Delta \omega, \kappa_{0}+\Delta \kappa\right) \\
= & M_{(t+\Delta t)}{ }^{-1} \cdot M_{(s+\Delta s)} \\
= & M_{\Delta t}{ }^{-1} \cdot\left(M_{t}^{-1} \cdot M_{s}\right) \cdot M_{\Delta s} \\
= & M_{\Delta t}{ }^{-1} \cdot M_{s}^{t} \cdot M_{\Delta s}
\end{aligned}
$$

Here, $M_{s}^{t}$ is obtained using (2):

$M_{\Delta t}=\left[\begin{array}{ccc}\cos \Delta \gamma_{t} \cos \Delta \alpha_{t}-\sin \Delta \gamma_{t} \sin \Delta \beta_{t} \sin \Delta \alpha_{t} & \sin \Delta \gamma_{t} \cos \Delta \alpha_{t}+\cos \Delta \gamma_{t} \sin \Delta \beta_{t} \sin \Delta \alpha_{t} & -\cos \Delta \beta_{t} \sin \Delta \alpha_{t} \\ -\sin \Delta \gamma_{t} \cos \Delta \beta_{t} & \cos \Delta \gamma_{t} \cos \Delta \beta_{t} & \sin \Delta \beta_{t} \\ \cos \Delta \gamma_{t} \sin \Delta \alpha_{t}+\sin \Delta \gamma_{t} \sin \Delta \beta_{t} \cos \Delta \alpha_{t} & \sin \Delta \gamma_{t} \sin \Delta \alpha_{t}-\cos \Delta \gamma_{t} \sin \Delta \beta_{t} \cos \Delta \alpha_{t} & \cos \Delta \beta_{t} \cos \Delta \alpha_{t}\end{array}\right]$

$M_{\Delta s}=\left[\begin{array}{ccc}\cos \Delta \gamma_{s} \cos \Delta \alpha_{s}-\sin \Delta \gamma_{s} \sin \Delta \beta_{s} \sin \Delta \alpha_{s} & \sin \Delta \gamma_{s} \cos \Delta \alpha_{s}+\cos \Delta \gamma_{s} \sin \Delta \beta_{s} \sin \Delta \alpha_{s} & -\cos \Delta \beta_{s} \sin \Delta \alpha_{s} \\ -\sin \Delta \gamma_{s} \cos \Delta \beta_{s} & \cos \Delta \gamma_{s} \cos \Delta \beta_{s} & \sin \Delta \beta_{s} \\ \cos \Delta \gamma_{s} \sin \Delta \alpha_{s}+\sin \Delta \gamma_{s} \sin \Delta \beta_{s} \cos \Delta \alpha_{s} & \sin \Delta \gamma_{s} \sin \Delta \alpha_{s}-\cos \Delta \gamma_{s} \sin \Delta \beta_{s} \cos \Delta \alpha_{s} & \cos \Delta \beta_{s} \cos \Delta \alpha_{s}\end{array}\right]$

where $\Delta \beta_{t}, \Delta \alpha_{t}$ and $\Delta \gamma_{t}$ are the rotation angles of the optical axis of the camera around the $X_{t}, Y_{t}$ and $Z_{t}$ axis, respectively, and $\Delta \beta_{s}, \Delta \alpha_{s}$ and $\Delta \gamma_{s}$, are the changes in the rotation angle of the optical axis of the star sensor around the $X_{s}, Y_{s}$, and $Z_{s}$ axis, respectively. All of them can be calculated using Table 1 , then the angular variation $(\Delta \varphi \quad \Delta \omega \quad \Delta \kappa)$ between the star and earth cameras can be solved.

According to the accuracy verification performed by (LIU et al., 2018), $\Delta \beta$ and $\Delta \alpha$ are insignificant, i.e., $\sin \Delta \beta=\sin \Delta \alpha \approx 0$ and $\cos \Delta \beta=\cos \Delta \alpha \approx 1$. After simplifying (7) and (8), it can be seen that the calibration accuracy of the angle between the optical axes of the cameras is approximately the same as that of a single camera.

\section{CONCLUSION}

Based on the attitude measurement principle of the star and earth cameras, long-term data was used to statistically analyze the main 
error sources that restrict the positioning accuracy. The angle variation between the star sensor's optical axis and the earth observation camera's optical axis caused by thermos-elastic error is shown to affect the accuracy of satellite positioning. To improve the positioning accuracy, a calibration method for the optical camera angle is proposed based on the optical autocollimation principle. The proposed method can monitor the changes of angles between the star sensor's optical axis and earth camera's optical axis without depending on the ground conditions or the original optical path. The experimental results demonstrated that the calibration accuracy of the angle between the optical axes of the cameras is approximately the same as that of a single camera, that is $\Delta \varphi$ and $\Delta \omega$ are better than $0.1^{\prime \prime}$, and $\Delta \kappa$ is about $2^{\prime \prime}$.

\section{REFERENCES}

Amberg V, Dechoz C, Bernard L, et al., 2013. In-flight attitude perturbances estimation: application to PLEIADES-HR satellites. Proc Spie, 8866(2):12.

Blarre L, Ouaknine J, Oddosmarcel L, et al., 2006, High Accuracy Sodern Star Trackers: Recent Improvements Proposed on SED36 and HYDRA Star Trackers. Aiaa Guidance, Navigation, \&Control Conference \& Exhibit.

Chen Y, Xie Z, Qiu Z, et al., 2015. Calibration and Validation of ZY-3 Optical Sensors. IEEE Transactions on Geoscience \& Remote Sensing, 53(8):4616-4626.

Delabie T, Schutter J D, Vandenbussche B., 2014. An Accurate and Efficient Gaussian Fit Centroiding Algorithm for Star Trackers. The Journal of the Astronautical Sciences, 61(1):60-84

ECSS Secretariat., 2008. ECSS-E-ST-60-20C:stars sensors terminology and performance specification. Noordwijk: ESA.

Erwin R, Babuska V, Sullivan L, et al., 2013. On-Orbit Active Vibration Isolation: The Satellite Ultraquiet Isolation Technologies Experiment (SUITE). Aiaa Space Conference \& Exposition.

Fausz J, Wilson B, Hall C, et al., 2012. Survey of Technology Developments in Flywheel Attitude Control and Energy Storage Systems. Journal of Guidance Control \& Dynamics, 33(1):286286.

Hashimoto T, Hamasaki T, Nakatani I, et al., 2013. Attitude control system design of a satellite with a magnetically suspended momentum wheel based on two-degree-of-freedom control system theory. Aiaa Guidance, Navigation \& Control Conference.

Iwata T, Hoshino H, Yoshizawa T, et al., 2007. Precision Attitude Determination for the Advanced Land Observing Satellite (ALOS): Design, Verification, and On-Orbit Calibration. AIAA Guidance, Navigation and Control Conference and Exhibit.

JIANG Y H, ZHANG G, TANG X, et al., 2014. Detection and Correction of Relative Attitude Errors for ZY1-02C. IEEE Transactions on Geoscience and Remote Sensing, 52(12):76747683.

LI S. M., LI Y., 2012, Mapping Satellite-1 transmission type photogrammetric and remote sensing satellite. Journal of Remote Sensing.
LIU H. B., HUANG S. H., TAN C. J., et al., 2009. Thermo-optic Effects on Accuracy Performance of Star Tracker. Acta Photonica Sinica, 38(7):1835-1839.

LIU W., WANG H., LIU D., et al., 2018. Exploiting AutoCollimation for Real-Time Onboard Monitoring of Space Optical Camera Geometric Parameters. ISPRS Annals of Photogrammetry, Remote Sensing \& Spatial Information Sciences, Vol. 4 Issue 2, p217-222. 6p.

LU X., WU Y. P., ZHONG H. J., et al., 2014. Low Frequency Error Analysis of Star Sensor. Aerospace Control \& Application.

Padgett C, Kreutz-Delgado K, Udomkesmalee S., 2012. Evaluation of Star Identification Techniques. Journal of Guidance Control \& Dynamics, 20(2):259-267.

Psiaki, Mark L., 2000. Attitude-Determination Filtering via Extended Quaternion Estimation. Journal of Guidance, Control, and Dynamics, 23(2):206-214.

QIAO P. Y., HE X., WEI Z. H., 2012. Application of weighted total least squares in measurement of deflection angle with optical self-collimation method. Optics and Precision Engineering, 20(9):1953-1959.

Radhadevi P V, Rupert Müller, D'Angelo P, et al., 2011. In-flight Geometric Calibration and Orientation of ALOS/PRISM Imagery with a Generic Sensor Model. Photogrammetric Engineering and Remote Sensing, 77(2011-05):531-538.

TING S., FEI X., ZHENG Y., 2013. Optical System Error Analysis and Calibration Method of High-Accuracy Star Trackers. Sensors, 13(4):4598-4623.

WANG H., LIU W., YU J. D., et al., 2018. Geometric Parameters Monitoring Technology for Space Optical Camera. ACTA PHOTONICASINICA, 47(10)

WANG M., FAN C., PAN J., et al., 2017. Image jitter detection and compensation using a high-frequency angular displacement method for Yaogan-26 remote sensing satellite. ISPRS Journal of Photogrammetry and Remote Sensing, 130:32-43.

WANG, R. X., 2004. The difficult problem about satellite photogrammetry without con-trol points. Science of Surveying \& Mapping, 29(3):3-5.

Wang R. X, HU X., 2012. The construction and application of Mapping Satellite-1 engineering. Journal of Remote Sensing.

WANG X. T., LI Y. C., 2012. Mapping Satellite-1 star sensor accuracy analysis. Journal of Remote Sensing.

WANG Z. Z., 1990. Principle of Photogrammetry. Beijing, Publishing House of Surveying and Mapping.

Winkler S, Wiedermann G, Gockel W., 2008. High-Accuracy On-Board Attitude Estimation for the GMES Sentinel-2 Satellite: Concept, Design, and First Results. AIAA Guidance, Navigation and Control Conference and Exhibit.

XU B., LEI B., FAN C. C., et al., 2016. Internal Error Compensation Method Based on High-Frequency Angular Displacement for High-Resolution Optical Satellite Images. Acta Optica Sinica, 36(9):0928002. 